\title{
Inhibidores de proteínas JAK (JAKi) en enfermedades autoinmunes
}

\section{JAK protein inhibitors (JAKi) in autoimmune diseases}

\author{
Juan A. Suárez-Cuenca* y Amy R. Rojas-Noverón
}

Laboratorio de Metabolismo Experimental e Investigación Clínica, Centro Médico Nacional 20 de Noviembre, Instituto de Seguridad y Servicios Sociales de los Trabajadores del Estado (ISSSTE), Ciudad de México, México

\section{Resumen}

Los inhibidores de las proteínas JAK (JAKi) son un nuevo grupo de fármacos prometedores desarrollados a raíz del entendimiento más profundo de los mecanismos fisiopatológicos de las enfermedades autoinmunes y proinflamatorias, dirigidos a la modulación de citocinas y moléculas que son parte de vías de señalización intracelular. Estos actúan directamente en la modulación del proceso inflamatorio a través de su interacción con receptores I y II de citocinas. Los JAKi se han propuesto como inmunomoduladores para pacientes con enfermedades autoinmunes o proinflamatorias en estadios severos, ylo que no responden a los tratamientos de primeras líneas como: Artritis reumatoide, artritis psoriásica, colitis ulcerativa, policitemia vera, mielofibrosis, neumonía atípica por Covid-19, entre otras, en las que actualmente se está probando su eficacia en ensayos clínicos. Entre las implicaciones clínicas de los JAKi destacan su papel en el sistema hematopoyético, el sistema inmunológico, y las funciones vitales en el desarrollo y supervivencia en la etapa embriológica. Por lo que hemos de permanecer alertas hasta definir su potencial terapéutico y su perfil de seguridad a largo plazo.

Palabras clave: Inhibidores de Jak. Cinasa Janus. JAK. Enfermedades autoinmunes. Nuevo tratamiento. COVID-19. Artritis reumatoide.

\begin{abstract}
JAK protein inhibitors (JAKi) are a promising new group of drugs developed because of a deeper understanding of the pathophysiological mechanisms of autoimmune and pro-inflammatory diseases, targeting the modulation of cytokines and molecules that are part of intracellular signaling pathways. These act directly in the modulation of the inflammatory process through their interaction with receptors I and II of cytokines. JAKi have been proposed as immunomodulators for patients with autoimmune or pro-inflammatory diseases in severe stages, and / or that do not respond to first-line treatments such as: rheumatoid arthritis, psoriatic arthritis, ulcerative colitis, polycythemia vera, myelofibrosis, atypical pneumonia due to Covid -19, among others, which are currently being tested for efficacy in clinical trials. Among the clinical implications of JAKi are their role in the hematopoietic system, the immune system, and vital functions in development and survival in the embryological stage. Therefore, we must remain vigilant until its therapeutic potential and its long-term safety profile are defined.
\end{abstract}

Keywords: JAK inhibitors. Janus kinase. JAK. Autoimmune diseases. New treatment. COVID-19. Rheumatoid arthritis.

Correspondencia:

*Juan A. Suárez-Cuenca

E-mail: suarej05@gmail.com
Disponible en internet: 22-02-2022

Fecha de aceptación: 03-11-2021

DOI: 10.24875/IMIDS.M21000014
Rev Mex Enferm Inflam Inmunomed 2021;95(4):107-111

www.IMIDsMexico.com

2696-6867 / @ 2021 Permanyer. Éste es un artículo open access bajo la licencia CC BY-NC-ND (http://creativecommons.org/licenses/by-nc-nd/4.0/). 


\section{Introducción}

Gracias al avance de los recursos tecnológicos y las investigaciones traslacionales precedentes se han podido comprender con mayor profundidad los mecanismos fisiopatológicos de las enfermedades autoinmunes y proinflamatorias. Con base en este nuevo conocimiento se han desarrollado nuevos medicamentos más específicos, dirigidos a la modulación de citocinas y moléculas que son parte de vías de señalización intracelular.

\section{Mecanismo de acción de JAK}

Una de las estrategias emergentes más prometedoras es la regulación de la actividad de proteínas JAK (cinasa de la proteína Janus, por sus siglas en inglés Janus Kinase), mediante el uso de inhibidores específicos (JAKi). Las JAK son una familia de cuatro proteínas del grupo de las tirosina cinasas: JAK1, JAK2, JAK3 y TYK2, que actúan directamente en la modulación del proceso inflamatorio a través de su interacción con los receptores I y II de citocinas (Fig. 1). Tras la unión del receptor de citocinas con su ligando, las proteínas JAK pueden autofosforilarse o transfosforilar moléculas intracelulares, iniciando una cascada de señalización o favoreciendo la dimerización de activadores de la transcripción (STAT), lo cual permite su activación y translocación al núcleo, culminando en un efecto regulador positivo o negativo sobre la transcripción génica ${ }^{1-3}$.

\section{Implicaciones de la proteína JAK}

Estudios previos han señalado la importancia de las proteínas JAK en diversas funciones, destacando su papel en el sistema hematopoyético. La mutación V617F con ganancia de función de JAK2 induce proliferación independiente de eritropoyetina y factores de crecimiento hematopoyético, resultando en policitemia vera, trombocitemia esencial y mielofibrosis en humanos ${ }^{4}$. El ruxolitinib, un inhibidor de JAK2 ya aprobado por la Food and Drug Administration (FDA), resultó ser ideal para tratar estas patologías hematológicas, como se detalla en la tabla 1.

Por otro lado, las proteínas JAK también están involucradas en la respuesta inmunitaria. Esto se vuelve evidente al analizar los efectos adversos de los JAKi, que son similares a los observados en las mutaciones con pérdida de función de JAK. Por ejemplo, se han descrito hasta 34 mutaciones en el gen JAK3, de herencia autosómica recesiva, asociadas con el síndrome de inmunodeficiencia combinada, una patología caracterizada por la ausencia de linfocitos T maduros y células NK circulantes, linfocitos B no funcionales en cantidades normales o elevadas, y una marcada hipoplasia de tejidos linfoides ${ }^{5}$. Los pacientes con síndrome de inmunodeficiencia combinada son altamente susceptibles a infecciones por bacterias, hongos y virus, al igual que los efectos colaterales de JAKi.

Al parecer, los JAK también cumplen funciones vitales durante la embriogénesis, ya que las mutaciones de pérdida de función de JAK1/2 son incompatibles con la vida en modelos múridos, mientras que en humanos no se han descrito.

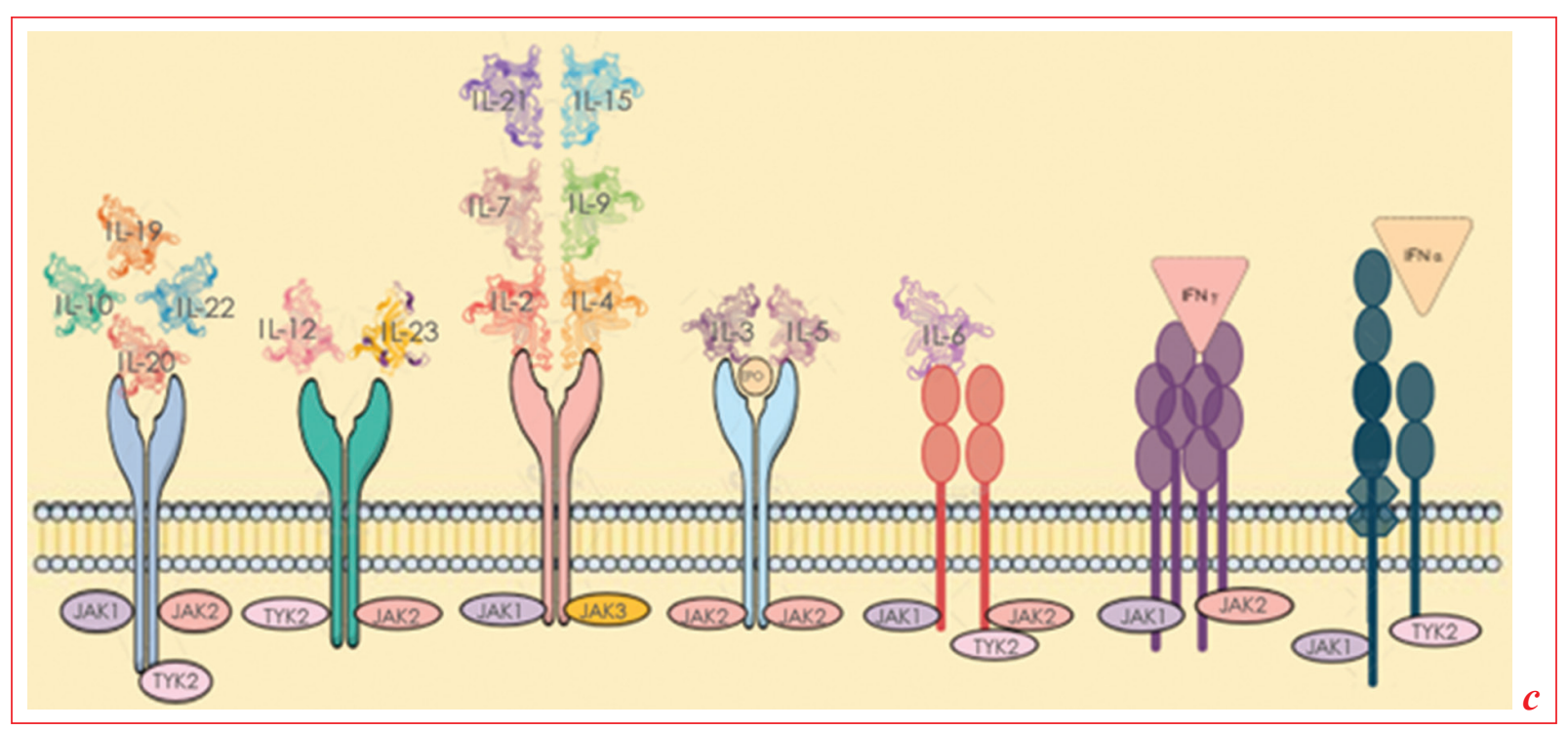

Figura 1. Selectividad de diversas citocinas hacia los receptores de citocinas tipo I y II dependientes de JAK. 
Tabla 1. Usos aprobados para los JAKi

\begin{tabular}{|c|c|c|c|c|c|}
\hline $\begin{array}{l}\text { Medicamento } \\
\text { (nombre } \\
\text { comercial) }\end{array}$ & $\begin{array}{l}\text { JAK de } \\
\text { afinidad }\end{array}$ & $\begin{array}{l}\text { Fecha de } \\
\text { aprobación } \\
\text { por la FDA }\end{array}$ & Aplicaciones & Dosis & $\begin{array}{l}\text { Precauciones y } \\
\text { efectos adversos }\end{array}$ \\
\hline $\begin{array}{l}\text { Tofacitinib } \\
\text { (Xeljanz, } \\
\text { Xeljanz XR) }\end{array}$ & $\begin{array}{l}\text { JAK } 1 \\
\text { JAK } 3\end{array}$ & 2017 & $\begin{array}{l}\text { Artritis reumatoide, } \\
\text { artritis psoriásica, } \\
\text { colitis ulcerativa }\end{array}$ & $\begin{array}{l}\text { AR: XELJANZ } 5 \mathrm{mg} \mathrm{c} / 12 \mathrm{~h} \text { o } \\
\text { XELJANZ XR } 11 \mathrm{mg} \text { c/24 h } \\
\text { PsoAR: mismas dosis, + } \\
\text { DMARD no biológicos } \\
\text { CoU: XELJANZ } 10 \mathrm{mg} \text { c/12 al } \\
\text { menos } 8 \text { semanas, después } 5 \text { o } \\
10 \mathrm{mg} \text { c/12 } \mathrm{h} \\
\text { Descontinuar si después de } 16 \\
\text { semanas con } 10 \mathrm{mg} \mathrm{c} / 12 \mathrm{~h} \text { no } \\
\text { se alcanza el beneficio } \\
\text { terapéutico } \\
\text { Usar la menor dosis } \\
\text { respuesta-efectiva }\end{array}$ & $\begin{array}{l}\text { Riesgo incrementado } \\
\text { de presentar } \\
\text { problemas } \\
\text { relacionados con el } \\
\text { corazón y cáncer } \\
\text { No usar si } \mathrm{CL}<500 \\
\mathrm{cel} / \mathrm{mm}^{3}, \mathrm{ANC}<1000 \\
\mathrm{cel} / \mathrm{mm}^{3} \text { o } \mathrm{Hb}<9 \mathrm{~g} / \mathrm{dl}\end{array}$ \\
\hline $\begin{array}{l}\text { Baricitinib }^{8} \\
\text { (Olumiant) }\end{array}$ & $\begin{array}{l}\text { JAK } 1 \\
\text { JAK2 }\end{array}$ & $\begin{array}{l}\text { NCB28050, } \\
2018\end{array}$ & Artritis reumatoide & $\begin{array}{l}2 \mathrm{mg} \mathrm{c} / 24 \text { h vía oral } \\
\text { Modificar dosis en enfermedad } \\
\text { renal, hepática, hematológica e } \\
\text { infecciones }\end{array}$ & $\begin{array}{l}\text { Riesgo incrementado } \\
\text { de problemas } \\
\text { cardiacos y cáncer }\end{array}$ \\
\hline $\begin{array}{l}\text { Ruxolitinib10,11 } \\
\text { (Jakafi) }\end{array}$ & $\begin{array}{l}\text { JAK } 1 \\
\text { JAK2 }\end{array}$ & $\begin{array}{l}\text { COMFORT-I, } \\
2012 . \\
\text { REACH-3, } 22 \\
\text { septiembre } \\
2021\end{array}$ & $\begin{array}{l}\text { Mielofibrosis, } \\
\text { policitemia vera, } \\
\text { GvHD aguda y } \\
\text { crónica, después de } \\
\text { fallar } 1 \text { o } 2 \text { líneas de } \\
\text { tratamiento } \\
\text { sistémico en } \\
\text { pacientes }>12 \text { años }{ }^{5}\end{array}$ & $\begin{array}{l}\text { MF: dependiendo de la CP, de } \\
5-20 \mathrm{mg} \text { c/12 h vía oral } \\
\text { PV: } 10 \mathrm{mg} \mathrm{c} / 12 \mathrm{~h} \text { vía oral } \\
\text { GvHD: } 5 \mathrm{mg} \mathrm{c} / 12 \mathrm{~h} \text { vía oral }\end{array}$ & $\begin{array}{l}\text { Trombocitopenia, } \\
\text { anemia, petequias, } \\
\text { equimosis, mareo, } \\
\text { cefalea, diarrea } \\
\text { Neutropenia, edema, e } \\
\text { infecciones }\end{array}$ \\
\hline $\begin{array}{l}\text { Fedratinib }{ }^{12} \\
\text { (Inrebic) }\end{array}$ & JAK 2 & $\begin{array}{l}\text { JAKARTA, } \\
\text { agosto } 2019\end{array}$ & $\begin{array}{l}\text { MF primaria o } \\
\text { secundaria } \\
\text { intermedia-2 o de } \\
\text { alto riesgo } \\
\text { (pospolicitemia vera } \\
0 \\
\text { postrombocitopenia } \\
\text { esencial) }\end{array}$ & $\begin{array}{l}\text { Pacientes con CP } \geq 50 \times 10^{9} / \mathrm{l} \text { : } \\
400 \mathrm{mg} \text { c/24 h vía oral } \\
\text { Ajustar dosis en pacientes con } \\
\text { inhibidores de CYP3A o función } \\
\text { renal disminuida }\end{array}$ & $\begin{array}{l}\text { Encefalopatía grave y } \\
\text { fatal, incluyendo } \\
\text { enfermedad de } \\
\text { Wernicke } \\
\text { Anemia y } \\
\text { trombocitopenia } \\
\text { Toxicidad } \\
\text { gastrointestinal y } \\
\text { hepatotoxicidad } \\
\text { Elevación de amilasa y } \\
\text { lipasa }\end{array}$ \\
\hline $\begin{array}{l}\text { Upadacitinib } \\
\text { (Rinvoq) }^{14}\end{array}$ & $\begin{array}{l}\text { JAK } 1 \\
\text { JAK2 }\end{array}$ & $\begin{array}{l}\text { SELECT } \\
2019\end{array}$ & $\begin{array}{l}\text { AR activa, moderada } \\
\text { a grave, que haya } \\
\text { tenido una respuesta } \\
\text { inadecuada o } \\
\text { intolerancia a } \\
\text { metotrexato }\end{array}$ & $\begin{array}{l}15 \mathrm{mg} \mathrm{c} / 24 \text { h vía oral como } \\
\text { monoterapia o en conjunto con } \\
\text { un DMARD no biológico } \\
\text { Suspender si } \mathrm{CL}<500 \mathrm{cel} / \mathrm{mm}^{3} \text {, } \\
\text { ANC }<1000 \mathrm{cel} / \mathrm{mm}^{3} \\
\text { o } \mathrm{Hb}<8 \mathrm{~g} / \mathrm{dl}\end{array}$ & $\begin{array}{l}\text { Infecciones graves, } \\
\text { malignidad y trombosis } \\
\text { Perforación } \\
\text { gastrointestinal } \\
\text { Embriotoxicidad y } \\
\text { fetotoxicidad, evitar su } \\
\text { uso en conjunto con } \\
\text { vacunas de virus vivos }\end{array}$ \\
\hline
\end{tabular}

ANC: conteo absoluto de neutrófilos; AR: artritis reumatoide; CL: cuenta leucocitaria; CoU: colitis ulcerativa; CP: cuenta plaquetaria; DMARD: fármacos modificadores de la enfermedad (disease modifying antirheumatic drugs); FDA: Food and Drug Administration; GvHD: enfermedad del injerto contra el huésped (graft-versus-host-disease); $\mathrm{Hb}$ : hemoglobina; MF: mielofibrosis; PsoAR: artritis psoriásica; PV: policitemia vera.

\section{JAKi}

Los JAKi no se consideran terapias biológicas; se refieren a moléculas inhibidoras específicas de la familia de proteínas JAK. La primera generación de JAKi se caracteriza por su capacidad de inhibir de tres a cuatro JAK, y también se denominan pan-JAKi. Un ejemplo es el peficitinib (Smyraf ${ }^{\circledR}$, Astellas Pharma), un medicamento aprobado en Japón (pero aún no por la FDA) para el tratamiento de la artritis reumatoide, capaz de inhibir JAK $1 / 2 / 3$ y TYK2 ${ }^{6}$. A diferencia de este, las nuevas generaciones de JAKi buscan tener mayor selectividad por cierto JAK, con el fin de mitigar los efectos adversos no deseados?

En general, la administración de los JAKi puede ser por vía tópica u oral, y algunos incluso cuentan con una presentación de liberación retardada. Dichas propiedades provocan menos efectos adversos en comparación con el uso de corticosteroides tópicos, como atrofia 
Tabla 2. Ensayos clínicos en curso

\begin{tabular}{|c|c|c|c|c|c|}
\hline $\begin{array}{l}\text { Medicamento } \\
\text { (nombre } \\
\text { comercial) }\end{array}$ & Estudio clínico & $\begin{array}{l}\text { Resultados en estudio } \\
\text { clínico }\end{array}$ & Aplicación & Dosis & $\begin{array}{l}\text { Precaución y } \\
\text { efectos adversos }\end{array}$ \\
\hline $\begin{array}{l}\text { Upadacitinib } \\
\text { (Rinvoq) }^{13-16}\end{array}$ & $\begin{array}{l}\text { U-ACCOMPLISH } \\
\text { (fase 3), enero } \\
2021\end{array}$ & $\begin{array}{l}33 \% \text { de los pacientes } \\
\text { alcanzaron la primera } \\
\text { meta de remisión clínica y } \\
\text { se cumplieron todas las } \\
\text { metas secundarias } \\
\text { Los resultados de } \\
\text { seguridad fueron } \\
\text { consistentes con la fase } \\
\text { de inducción previa, no } \\
\text { se observaron nuevos } \\
\text { riesgos para la salud }\end{array}$ & Colitis ulcerativa & $\begin{array}{l}45 \mathrm{mg} \mathrm{c} / 24 \mathrm{~h} \\
\text { vía oral }\end{array}$ & $\begin{array}{l}\text { Alto riesgo de } \\
\text { malignidad, } \\
\text { infecciones graves } \\
\text { y trombosis }\end{array}$ \\
\hline $\begin{array}{l}\text { Baricitinib }^{9} \\
\text { (Olumiant) }\end{array}$ & $\begin{array}{l}\text { COV-BARRIER, } \\
28 \text { julio } 2021\end{array}$ & $\begin{array}{l}\text { Baricitinib resultó ser } \\
\text { igual de efectivo que } \\
\text { remdesivir, por lo que se } \\
\text { autoriza su uso como } \\
\text { monoterapia de } \\
\text { emergencia en COVID-19 }\end{array}$ & $\begin{array}{l}\text { Uso de emergencia } \\
\text { en COVID-19: } \\
\text { pacientes } \geq 2 \text { años } \\
\text { que requieran } 0_{2} \\
\text { suplementario, } \\
\text { ventilación mecánica } \\
\text { invasiva o no } \\
\text { invasiva, o ECM0, } \\
\text { que cumplan con las } \\
\text { autorizaciones del } \\
\text { acto }\end{array}$ & $\begin{array}{l}4 \mathrm{mg} \mathrm{c} / 24 \text { h vía } \\
\text { oral }\end{array}$ & $\begin{array}{l}\text { Riesgo } \\
\text { incrementado de } \\
\text { problemas } \\
\text { cardiacos, cáncer, } \\
\text { alteraciones } \\
\text { hematológicas y } \\
\text { hepatotoxicidad }\end{array}$ \\
\hline Itacitinib ${ }^{17}$ & $\begin{array}{l}\text { INCB039110 } \\
\text { (fase 2) }\end{array}$ & $\begin{array}{l}\text { Itacitinib en combinación } \\
\text { con nab-paclitaxel }+ \\
\text { gemcitabina demostró un } \\
\text { perfil de seguridad } \\
\text { aceptable }\end{array}$ & $\begin{array}{l}\text { Actividad clínica } \\
\text { aceptable en } \\
\text { pacientes con } \\
\text { tumores sólidos } \\
\text { avanzados, incluido } \\
\text { cáncer pancreático }\end{array}$ & $400 \mathrm{mg} \mathrm{c} / 24 \mathrm{~h}$ & $\begin{array}{l}\text { Sangrado de tubo } \\
\text { digestivo } \\
\text { Gastrotoxicidad y } \\
\text { hepatotoxicidad }\end{array}$ \\
\hline Pacritinib ${ }^{18}$ & $\begin{array}{l}\text { PERSIST-1 } \\
\text { (fase 3) }\end{array}$ & $\begin{array}{l}\text { Bien tolerado, indujo una } \\
\text { reducción significativa y } \\
\text { sostenida del volumen } \\
\text { esplénico, y reducción de } \\
\text { los síntomas incluso en } \\
\text { pacientes con citopenias } \\
\text { graves }\end{array}$ & $\begin{array}{l}\text { Mielofibrosis de alto } \\
\text { riesgo }\end{array}$ & $400 \mathrm{mg} \mathrm{c} / 24 \mathrm{~h}$ & $\begin{array}{l}\text { Anemia, } \\
\text { insuficiencia } \\
\text { cardiaca, pirexia y } \\
\text { neumonía }\end{array}$ \\
\hline
\end{tabular}

ECMO: oxigenación por membrana extracorpórea (extracorporeal membrane oxygenation).

local y telangiectasias, por lo que se está investigando su uso en dermatología para tratar otras enfermedades autoinmunes ${ }^{1}$. En la tabla 1 se resumen las principales aplicaciones clínicas ya aprobadas por la FDA, tales como artritis reumatoide, artritis psoriásica, colitis ulcerativa, policitemia vera, mielofibrosis, etc.

Sin embargo, su uso se extiende constantemente en otras enfermedades y modalidades de tratamiento, como se puede observar en los estudios que se realizan en la actualidad para el tratamiento de la colitis ulcerativa, neoplasias e incluso la neumonía atípica de la COVID-19 (Tabla 2).

Indudablemente, el uso de baricitinib como tratamiento de emergencia en la infección por SARS-CoV2 representa una de las aplicaciones más revolucionarias de los JAKi. Este inhibidor selectivo de JAK1/2 demostró buenos resultados en el estudio COV-BARRIER, un estudio clínico de fase 3 , doble ciego, aleatorizado y controlado con placebo, en el cual se evaluaron su eficacia y perfil de seguridad en combinación con el tratamiento estándar de pacientes hospitalizados por COVID-19. La meta primaria del estudio fue la proporción de progresión de la enfermedad a requerimientos de oxígeno de alto flujo, necesidad de ventilación no invasiva o ventilación mecánica invasiva, obteniendo una razón de momios de 0.85 (intervalo de confianza del 95\%: 0.67-1.08; $p=0.18$ ) y una reducción del riesgo absoluto de -2.7 con el uso de baricitinib. De manera 
secundaria se exploró la mortalidad al día 28 y al día 60 por cualquier causa. La reducción de la progresión de la enfermedad en el grupo de pacientes tratados con baricitinib no fue muy distinta de la del grupo con placebo, pero se demostró un perfil de seguridad similar al del tratamiento estándar por sí solo, con una asociación de mortalidad reducida con ambos puntos de corte, y también una menor frecuencia de efectos adversos graves en comparación con el grupo que recibió placebo ${ }^{10,19}$.

\section{Conclusiones}

Los JAKi son una estrategia terapéutica innovadora que proporciona alta selectividad para el control de vías de señalización. Representan también una nueva esperanza para los pacientes con enfermedades en estadios graves o que no responden a los tratamientos de primeras líneas.

A pesar de ser un blanco terapéutico prometedor para muchas enfermedades autoinmunes, la decisión de la FDA de no aprobar algunos JAKi, y la desistencia de algunos laboratorios por conseguir dicha aprobación, dejan muchas incógnitas sobre la mesa. En el caso del tofacitinib, la FDA no aprobó su uso para la psoriasis a pesar de demostrar buena eficacia en estudios de fase 3, por inconsistencias en su potencial terapéutico y su perfil de seguridad a largo plazo ${ }^{20}$.

Actualmente se continúa expandiendo la experiencia en enfermedades autoinmunes como la dermatitis atópica, el lupus eritematoso sistémico y la esclerosis sistémica, entre otras. Por ello, hemos de permanecer alertas a su eficacia y seguridad, por las implicaciones clínicas potenciales.

\section{Financiamiento}

Los autores declaran que no existe financiamiento o apoyo de cualquier otro tipo.

\section{Conflicto de intereses}

Ningún autor reportó conflictos de intereses potenciales.

\section{Responsabilidades éticas}

Protección de personas y animales. Los autores declaran que para esta investigación no se han realizado experimentos en seres humanos ni en animales.

Confidencialidad de los datos. Los autores declaran que en este artículo no aparecen datos de pacientes.
Derecho a la privacidad y consentimiento informado. Los autores declaran que en este artículo no aparecen datos de pacientes.

\section{Referencias}

1. Solimani F, Meier K, Ghoreschi K. Emerging topical and systemic JAK inhibitors in dermatology. Front Immunol. 2019;10:2847.

2. Fragoulis GE, McInnes IB, Siebert S. JAK-inhibitors. New players in the field of immune-mediated diseases, beyond rheumatoid arthritis. Rheumatology. 2019;58(Suppl 1):i43-54.

3. Banerjee S, Biehl A, Gadina M, Hasni S, Schwartz DM. JAK-STAT signaling as a target for inflammatory and autoimmune diseases: current and future prospects. Drugs. 2017;77(5):521-46.

4. Salhotra A, Oo TH. JAK2 (V617F) positive latent myeloproliferative neoplasm presenting with splanchnic vein thrombosis. Indian $\mathrm{J}$ Hematol Blood Transf. 2012;30(S1):4-8.

5. Shi P, Amin HM. JAK3 (Janus Kinase 3 or Just Another Kinase 3). atlasgeneticsoncology.org. 2017. Citado: 25/10/2021. Disponible en: http://atlasgeneticsoncology.org/Genes/JAK3ID41032ch19p13.html

6. Markham A, Keam SJ. Peficitinib: first global approval. Drugs. 2019;79:887-91.

7. Pfizer Inc, XELJANZ, XELJANZ XR (tofacitinib). U.S. Food and Drug Administration website. Citado: 20/10/2021. Disponible en: https://www.accessdata.fda.gov/drugsatfda_docs/label/2019/203214s024,208246s010lbl.pdf

8. Eli Lilly and Company. Olumiant (baricitinib). U.S. Food and Drug Administration website. Citado: 16/10/2021. Disponible en: https://www.accessdata.fda.gov/drugsatfda_docs/label/2020/207924s002lbl.pdf

9. Eli Lilly and Company. Olumiant (baricitinib). U.S. Food and Drug Administration website. Citado: 18/10/2021. Disponible en: https://www.fda.gov/ media/143825/download

10. Incyte Corp. JAKAFI (ruxolitinib). U.S. Food and Drug Administration website. Citado: 18/10/2021. Disponible en: https://www.fda.gov/drugs/ resources-information-approved-drugs/fda-approves-ruxolitinib-chronic-graft-versus-host-disease

11. Incyte Corp. JAKAFI (ruxolitinib). U.S. Food and Drug Administration website. Citado: 16/10/2021. Disponible en: https://www.accessdata.fda. gov/drugsatfda_docs/label/2020/202192Orig1s019Rpllbl.pdf

12. Celgene Corporation. INREBIC (fedratinib). U.S. Food and Drug Administration website. Citado: 19/10/2021. Disponible en: https:// www.accessdata.fda.gov/drugsatfda_docs/label/2019/212327s000lbl. pdf

13. Second phase 3 induction study confirms upadacitinib (RINVOQ ${ }^{T M}$ ) improved clinical, endoscopic and histologic outcomes in ulcerative colitis patients. AbbVie News Center. news.abbvie.com. 2021. Disponible en: https://news.abbvie.com/news/press-releases/second-phase-3-induction-study-confirms-upadacitinib-rinvoq-improved-clinical-endoscopic-and-histologic-outcomes-in-ulcerative-colitis-patients.htm

14. AbbVie. Rinvoq (upadacitinib). U.S. Food and Drug Administration website. Citado: 20/10/2021. Disponible en: https://www.accessdata.fda.gov/ drugsatfda_docs/label/2019/211675s000lbl.pdf

15. AbbVie. A multicenter, randomized, double-blind, placebo-controlled induction study to evaluate the efficacy and safety of upadacitinib (ABT-494) in subjects with moderately to severely active ulcerative colitis. clinicaltrials.gov. 2021 Citado: 25/10/2021. Disponible en: https://clinicaltrials.gov/ ct2/show/NCT03653026

16. Second phase 3 induction study confirms upadacitinib (RINVOQTM) improved clinical, endoscopic and histologic outcomes in ulcerative colitis patients. AbbVie News Center. news.abbvie.com. Disponible en: https://news.abbvie.com/news/press-releases/second-phase-3-induction-study-confirms-upadacitinib-rinvoq-improved-clinical-endoscopic-and-histologic-outcomes-in-ulcerative-colitis-patients.htm

17. Beatty GL, Shahda S, Beck T, Uppal N, Cohen SJ, Donehower R, et al. A phase $\mathrm{Ib} / \mathrm{ll}$ study of the JAK1 inhibitor, itacitinib, plus nab-paclitaxel and gemcitabine in advanced solid tumors. Oncologist. 2019;24(1):14-e10.

18. Mesa RA, Vannucchi AM, Mead A, Egyed M, Szoke A, Suvorov A, et al. Pacritinib versus best available therapy for the treatment of myelofibrosis irrespective of baseline cytopenias (PERSIST-1): an international, randomised, phase 3 trial. Lancet Haematol. 2017;4(5): e225-36.

19. Marconi VC, Ramanan AV, de Bono S, Kartman CE, Krishnan V, Liao R, et al. Efficacy and safety of baricitinib for the treatment of hospitalised adults with COVID-19 (COV-BARRIER): a randomised, double-blind, parallel-group, placebo-controlled phase 3 trial. Lancet Respir Med. 2021 Aug 31;S2213-2600(21)00331-3. doi: 10.1016/S22132600(21)00331-3. Online ahead of print.

20. Berekmeri A, Mahmood F, Wittmann M, Helliwell P. Tofacitinib for the treatment of psoriasis and psoriatic arthritis. Expert Rev Clin Immunol. 2018;14(9):719-30. 